\title{
Ptychographic Nano-Analytical Microscope (PtyNAMi) at PETRA III: signal-to-background optimization for imaging with high sensitivity
}

Christian G. Schroer, Martin Seyrich, Andreas Schropp, Ralph Döhrmann, Stephan Botta, et al.

Christian G. Schroer, Martin Seyrich, Andreas Schropp, Ralph Döhrmann, Stephan Botta, Patrik Wiljes, Dennis Brückner, Maik Kahnt, Felix Wittwer, Lukas Grote, Dorota Koziej, Jan Garrevoet, Gerald Falkenberg,

"Ptychographic Nano-Analytical Microscope (PtyNAMi) at PETRA III: signalto-background optimization for imaging with high sensitivity," Proc. SPIE 11112, X-Ray Nanoimaging: Instruments and Methods IV, 111120D (9 September 2019); doi: 10.1117/12.2529096

Event: SPIE Optical Engineering + Applications, 2019, San Diego, California, United States 


\title{
Ptychographic Nano-Analytical Microscope (PtyNAMi) at PETRA III: signal-to-background optimization for imaging with high sensitivity
}

\author{
Christian G. Schroer ${ }^{\mathrm{a}, \mathrm{b}}$, Martin Seyrich ${ }^{\mathrm{a}, \mathrm{b}}$, Andreas Schropp ${ }^{\mathrm{a}}$, Ralph Döhrmann ${ }^{\mathrm{a}}$, \\ Stephan Botta ${ }^{\mathrm{a}}$, Patrik Wiljes ${ }^{\mathrm{a}}$, Dennis Brückner ${ }^{\mathrm{a}, \mathrm{b}}$, Maik Kahnt ${ }^{\mathrm{a}, \mathrm{b}}$, Felix Wittwer ${ }^{\mathrm{a}, \mathrm{b}}$, \\ Lukas Grote $^{\mathrm{b}}$, Dorota Koziej ${ }^{\mathrm{b}}$, Jan Garrevoet ${ }^{\mathrm{a}}$, and Gerald Falkenberg ${ }^{\mathrm{a}}$

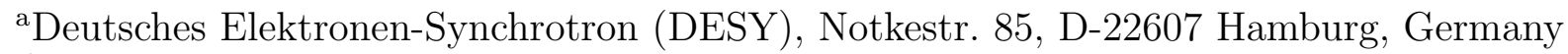

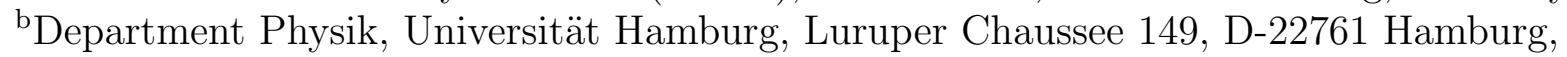 \\ Germany
}

\begin{abstract}
The X-ray scanning microscope PtyNAMi at beamline P06 of PETRA III at DESY in Hamburg, Germany, is designed for high-spatial-resolution 3D imaging with high sensitivity. Besides optimizing the coherent flux density on the sample and the precision mechanics of the scanner, special care has been taken to reduce background signals on the detector. The optical path behind the sample is evacuated up until the sensor of a four-megapixel detector that is placed into the vacuum. In this way, parasitic scattering from air and windows close to the detector is avoided. The instrument has been commissioned and is in user operation. The main commissioning results of the low-background detector system are presented. A signal-to-noise model for small object details is derived that includes incoherent background scattering.
\end{abstract}

Keywords: X-ray microscopy, ptychography, tomography, signal-to-background ratio, spatial resolution, detection sensitivity

\section{INTRODUCTION}

In recent years, ptychography has revolutionized X-ray microscopy in that it is able to overcome the diffraction limit of X-ray optics, ${ }^{1}$ pushing the spatial resolution limit down to a few nanometers. ${ }^{2,3}$ However, due to the weak interaction of X-rays with matter the detection of small features inside a sample requires a high coherent fluence, ${ }^{4}$ a high degree of mechanical stability, and a low background signal from the X-ray microscope. All these requirements need to be fulfilled simultaneously in order to be able to image small and weakly scattering objects at high resolution.

The hard X-ray scanning microscope PtyNAMi at beamline P06 of the synchrotron radiation source PETRA III at DESY in Hamburg, Germany, is designed in view of the above-mentioned requirements. It serves as a test bed for high-resolution and high-sensitivity imaging for the future upgrade of PETRA III to the ultralow emittance synchrotron radiation source PETRA IV ${ }^{5}$ and is continuously upgraded. The basic design was described in Ref. 6 with a focus on the mechanical stability of the instrument. In this article, we briefly review the instrument and focus on the effects of the instrumental scattering background on the image quality.

\section{PTYNAMI: THE HARD X-RAY NANOPROBE STATION AT PETRA III}

The hard X-ray nanoprobe station at beamline P06 in the Max von Laue Hall at PETRA III (electron energy: $6 \mathrm{GeV}$ ) was set up in 2010 and is in user operation since $2012 .^{7-9}$ During its operation many of the experimental requirements for high-resolution scanning microscopy and ptychography were elaborated. ${ }^{2,10}$ In 2015 , the Ptychographic Nano-Analytical Microscope (PtyNAMi) project was started as an upgrade to the hard X-ray nanoprobe. The scanner unit of this new instrument is located at $97 \mathrm{~m}$ from the undulator source (U32). The

Further author information: (Send correspondence to C. G. S.)

E-mail: christian.schroer@desy.de

\footnotetext{
X-Ray Nanoimaging: Instruments and Methods IV, edited by Barry Lai,

Andrea Somogyi, Proc. of SPIE Vol. 11112, 111120D · (c) 2019 SPIE

CCC code: $0277-786 X / 19 / \$ 21 \cdot$ doi: $10.1117 / 12.2529096$
} 


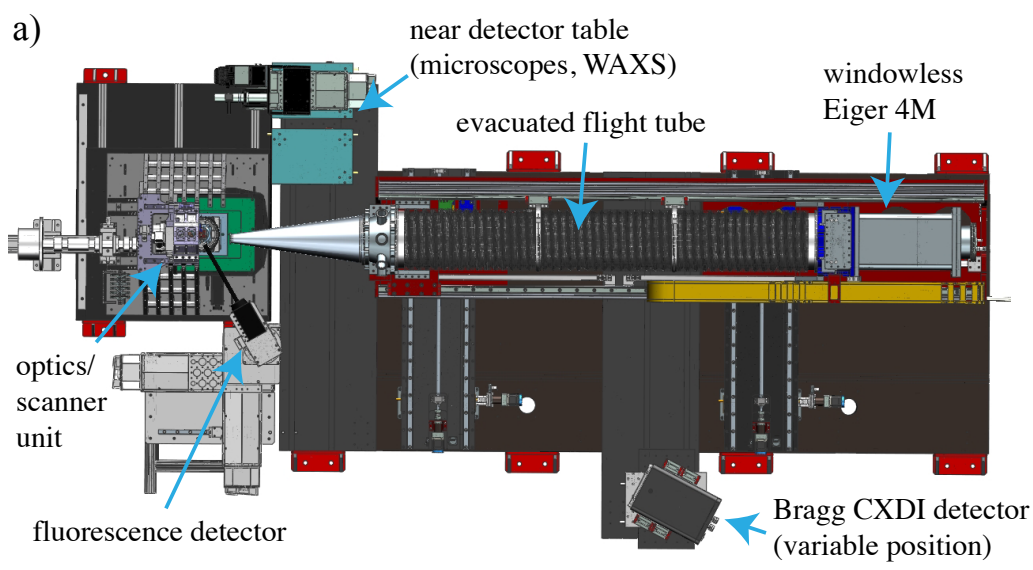

b)

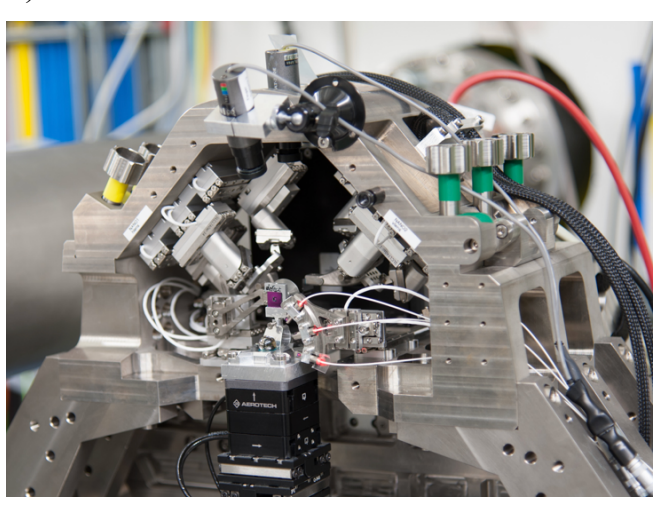

Figure 1. a) top view (CAD drawing) of the PtyNAMi end station at beamline P06 at PETRA III. b) Photograph of the scanner unit (tomography mode).

beam is monochromatized by a fixed-exit double-crystal or channel-cut monochromator (Si 111, vertical reflection). Higher harmonics are rejected by a double bounce total reflection mirror system (horizontal deflection). A subsequent prefocusing system made of a variable set of refractive lenses (automatic lens changer) is used to adjust the lateral coherence length on the entrance slit of the scanner unit (cf. Ref. 7 for the concept). Details about the beamline geometry and the components upstream of the scanner can be found in Ref. 9 .

The current design of the scanning microscope is shown in Figure 1. The new scanner unit has improved mechanical stability and positioning control compared to the previous design, ${ }^{9}$ implements efficient scanning schemes (e. g., fly scans), and allows for flexible and reliable alignment of various X-ray optics [nanofocusing and adiabatically focusing refractive X-ray lenses (NFLs/AFLs), ${ }^{11-14}$ Fresnel zone plates (FZP), and multilayer Laue lenses $(\mathrm{MLL})^{15}$ ]. There are two complementary sample scanners available, a very flexible one with tomographic scanning capability and a root-mean-square (rms) positioning stability of $\leq 10 \mathrm{~nm}$ and an ultra-stable setup for two-dimensional maps with $\leq 1 \mathrm{~nm}$ rms position stability. It is planned to replace the tomography scanner by one with improved positioning stability in the future.

The detector unit has also been completely replaced by the setup shown in Fig. 1a). The key feature is a flight tube with a windowless in-vacuum pixel detector (Eiger X 4M) for the detection of the (coherent) smallangle X-ray scattering (SAXS) signal for ptychographic imaging. The flight tube includes an optional in-vacuum beamstop to block the direct beam at about $35 \mathrm{~mm}$ before the detector. The sample-to-detector distance can be varied continuously between $1440 \mathrm{~mm}$ and $3340 \mathrm{~mm}$ to adapt the $q$ range for SAXS and ptychography. By changing the configuration of the bellows and vacuum tubes, other distances up to $4000 \mathrm{~mm}$ are achievable. The flight tube with the detector can be rotated around the sample position in the horizontal plane by maximally $20^{\circ}$ to detect high-resolution reciprocal space maps, ${ }^{16}$ e. g., for Bragg coherent X-ray diffraction imaging (Bragg CXDI) ${ }^{17}$ For the in-vacuum system, the angular range is limited due to space constraints inside the hutch. In order to reach higher diffraction angles up to $35^{\circ}$, the diffraction camera (e. g., Eiger X $4 \mathrm{M}$ ) can be placed on a variable mount as shown in Fig. 1a).

The nozzle of the flight tube can be retracted from the sample to make room for the detectors mounted on the near detector table. They are an in-line visible light microscope, a high-resolution X-ray camera, and a PIN diode. In addition, a pixel detector (e. g., Eiger X 4M) can be mounted on the near detector table for collecting wide-angle X-ray scattering (WAXS) data. There are free slots to position other detectors as needed. In order to record the fluorescence radiation from the sample, a silicon drift diode detector (Vortex EM) is positioned to face the sample from the side [cf. Fig. 1a)]. 
a)

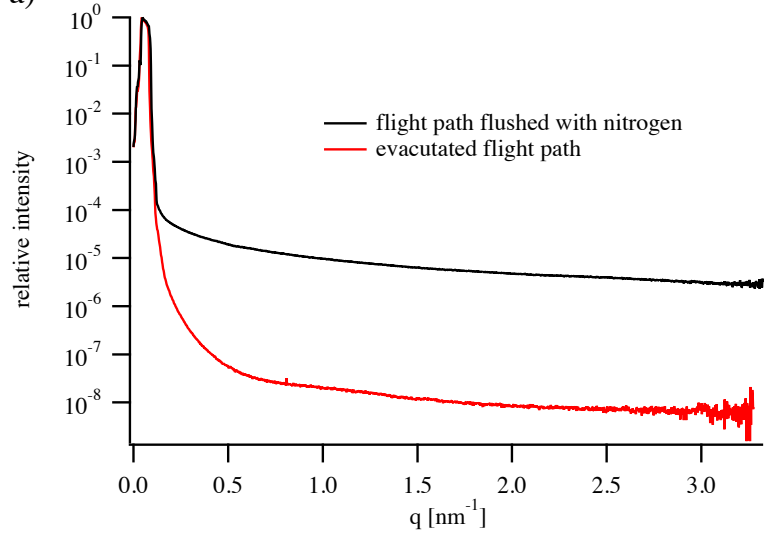

(2)

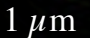

Figure 2. a) Beam intensity on the detector without any sample in the beam: azimuthal average for the case where the flight tube is flushed with nitrogen (black curve) and the evacuated case $\left(8 \times 10^{-4} \mathrm{mbar}\right.$, red curve). In this case, the coherent nanobeam was created by a Fresnel zone plate: b) complex wave field in the focal plane of the zone plate with central stop as determined by ptychography, c) far-field image of the nanobeam as measured on the pixel detector (cropped to $600 \times 600$ pixels around the optical axis).

\section{PTYCHOGRAPHIC IMAGING}

To record a ptychogram, the sample is scanned through the laterally confined coherent* hard X-ray beam with sufficient overlap of the illuminated areas of neighboring scan points. ${ }^{1,19}$ At each position of the scan, a far-field (SAXS) diffraction pattern is recorded. From these data, the complex transmission function and the complex illuminating wave field can be reconstructed. ${ }^{1}$ In the last decade, ptychography has made significant advances, combining it with tomography, ${ }^{20-22}$ absorption spectroscopic/resonant scattering contrast,${ }^{23-26}$ for characterizing X-ray optics and correcting aberrations. ${ }^{15,27-36}$

The main advantage of ptychography over conventional scanning microscopy is that its spatial resolution is not limited by the lateral size of the scanning beam but can be significantly higher. ${ }^{2}$ As for coherent X-ray diffraction imaging, the spatial resolution is limited by the highest momentum transfer $q$ to which the coherent far-field diffraction pattern can be recorded. Typically, the coherent diffraction signal of a non-periodic object decays strongly with increasing $q$ (i. e., $\propto q^{-\alpha}, \alpha \approx 4$ ). ${ }^{37}$ Ideally, with no instrumental background signal on the detector, the $q$ range in the diffraction pattern and thus the spatial resolution is limited by the photon statistics in the coherent diffraction pattern only. With an incoherent background signal present, however, the weak scattering signal at high $q$ can be covered up. In addition, the background is misinterpreted as coherent diffraction signal unless it is properly modelled and considered in the ptychographic reconstruction. ${ }^{38,39}$ In any case, the reconstruction suffers as compared to one from low-background data. ${ }^{3}$

In order to achieve highest sensitivity and resolution, it is thus favorable to reduce background scattering as much as possible in a ptychographic scanning microscope. Scattering in air or off a window close to the detector has the strongest contribution to an incoherent background signal, mainly because the solid angle spanned by the detector is large as seen from the position of the scattering event occurring close to the detector. In PtyNAMi, this is addressed by strongly reducing the number of scatterers in the beam path by placing the pixel detector in a vacuum $\left(p<1 \times 10^{-3}\right.$ mbar $)$ with no window in front of the sensor. The entrance window near the sample ( $>3 \mathrm{~m}$ away from the detector) can be a single-crystal diamond (thickness: $100 \mu \mathrm{m}$ ) or Kapton window (thickness: $25 \mu \mathrm{m})$.

Fig. 2a) shows the intensity distribution in the far field of the direct beam without any sample. Here, a Fresnel zone plate (diameter: $D=125 \mu \mathrm{m}$, outermost zone width $\Delta r_{n}=70 \mathrm{~nm}$, central stop diameter: $50 \mu \mathrm{m}$ )

\footnotetext{
*If the aperture of the nanofocusing optics is illuminated coherently, the focused beam is laterally coherent with the coherence length exceeding the beam size. ${ }^{18}$
} 

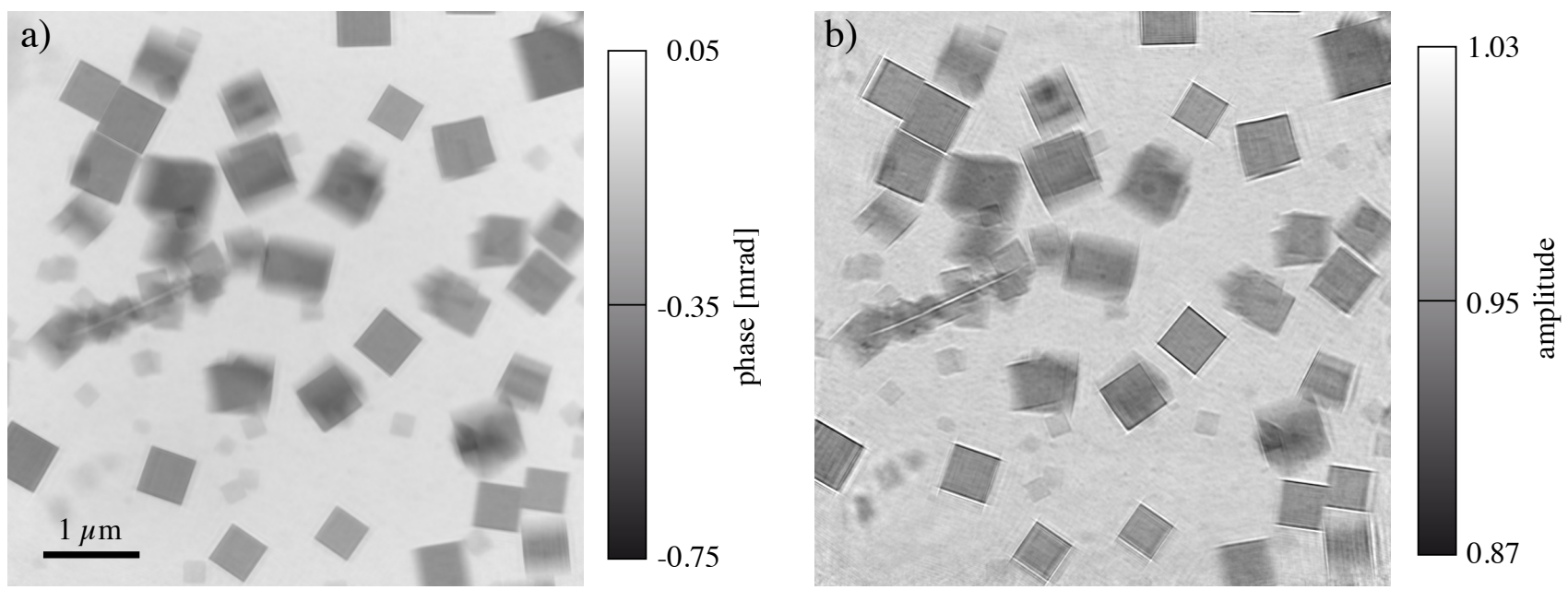

Figure 3. Ptychographic reconstruction of copper-(I)-oxide cubes and particles on a Kapton substrate: a) phase and b) amplitude. The $\mathrm{Cu}_{2} \mathrm{O}$ cubes were grown on the Kapton foil according to the synthesis protocol presented elsewhere. ${ }^{40,41}$

was used to generate the coherent nanobeam at an X-ray energy of $E=9.3 \mathrm{keV}$. The complex wave field as obtained by ptychography is shown in Fig. 2b) as well as the far-field image of the beam as measured by the Eiger X 4M detector (sample-position-to-detector distance: $3470 \mathrm{~mm}$ ) for an exposure time of $240 \mathrm{~s}$ in Fig. 2c).

In the case of a nitrogen-filled flight tube at ambient pressure, the background level lies between 4 and 5 orders of magnitude below the brightest parts of the beam [Fig. 2a)]. By evacuating the flight tube $\left(p=8 \times 10^{-4} \mathrm{mbar}\right)$, the background can be significantly reduced to the level of $1 \times 10^{-8}$ compared to the direct beam [Fig. 2a)].

As a result, clean ptychograms can be recorded with high resolution and sensitivity. Fig. 3 shows the ptychographic reconstruction of cubic copper-(I)-oxide particles on a Kapton substrate in phase and amplitude. The ptychogram was recorded about $900 \mu \mathrm{m}$ downstream of the focus of the above-mentioned Fresnel zone plate with X-rays of $E=9 \mathrm{keV}$ in a jittered grid scan of $20 \times 20$ steps of $250 \mathrm{~nm}$ and a dwell time of $2 \mathrm{~s}$. The Eiger $\mathrm{X} 4 \mathrm{M}$ was placed $3600 \mathrm{~mm}$ behind the sample. While the large cubes around $500 \mathrm{~nm}$ in size exhibit spatial resolution to the sampling limit (pixel size: $6.64 \mathrm{~nm}$ ), also smaller nanoparticles can be seen that are a few $10 \mathrm{~nm}$ in size and scatter more than three orders of magnitude less than the large cubes. The resolution for these small features is lower.

\section{SENSITIVITY VS. BACKGROUND}

In the following, we address the question of how the sensitivity of a ptychographic measurement depends on an incoherent background signal on the detector. Following Ref. 4, a detail or feature is considered separately from the rest of the object, and the question is addressed, under which conditions this feature can be detected. The diffraction pattern including an incoherent background signal can be modelled by

$$
P(\vec{q})=I_{c} \int_{\Delta \Omega}\left(\left|\psi_{d}(\vec{q})\right|^{2}+2 \Re\left(\psi_{d}(\vec{q}) \psi_{r}^{*}(\vec{q})\right)+\left|\psi_{r}(\vec{q})\right|^{2}+b(\vec{q})\right) d \Omega \cdot \Delta t
$$

where $P(\vec{q})$ is the number of photons in a pixel of the detector at the reciprocal space position $\vec{q}$ spanning the solid angle $\Delta \Omega$ and $\psi_{d}(\vec{q})$ and $\psi_{r}(\vec{q})$ are the complex amplitudes of the incident beam being scattered to $\vec{q}$ by the given feature or detail and the rest of the object, respectively. ${ }^{4} I_{c}$ is the coherent flux density on the sample and $\Delta t$ the exposure time. $b(\vec{q})$ is the (incoherent) background intensity.

For a small feature in a large sample, $\left|\psi_{d}\right|^{2} \ll\left|\psi_{r}\right|^{2}$, and the interference term $2 \Re\left(\psi_{d}(\vec{q}) \psi_{r}^{*}(\vec{q})\right)$ is the leading term in eq. (1) that carries information about the feature. Following a signal-to-background analysis similar to Ref. 4, this interference term lies a factor $\alpha$ (e. g., $\alpha=5$ to fulfil the Rose criterion) above the noise level of the rest of the sample and the background if the necessary condition

$$
I_{c} \Delta t \cdot\left|\psi_{d}\right|^{2} \Delta \Omega_{d} \geq \frac{\alpha^{2}}{4} \frac{\left\langle\left|\psi_{r}\right|^{2}\right\rangle+\langle b(\vec{q})\rangle}{\left\langle\left|\psi_{r}\right|^{2}\right\rangle} \rightarrow \frac{\alpha^{2}}{4} \quad \text { for }\left\langle\left|\psi_{r}\right|^{2}\right\rangle \gg\langle b(\vec{q})\rangle
$$




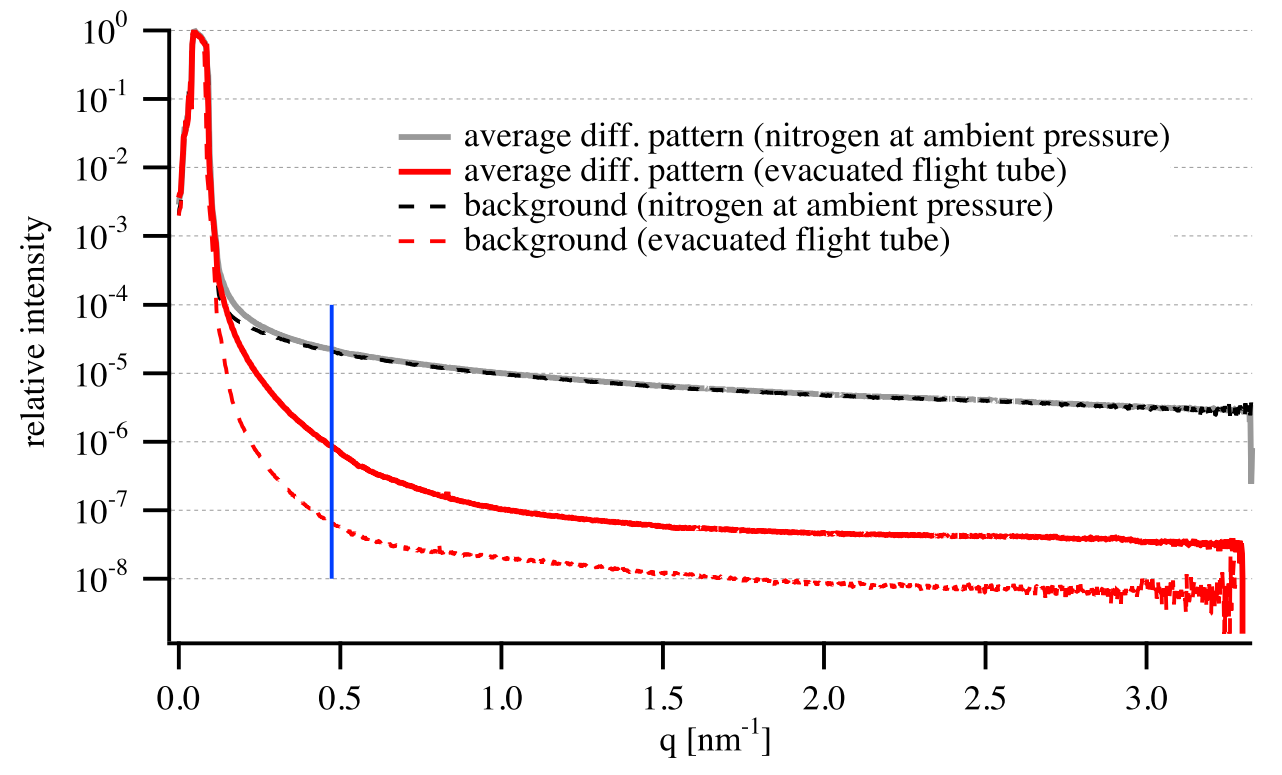

Figure 4. Azimuthal average over all diffraction patterns in the ptychogram recorded with evacuated flight tube as described in section 3 (red solid line) and a ptychogram recorded with similar parameters except for the flight tube filled with nitrogen at ambient pressure (gray solid line). For comparison, the azimuthal average of the far-field image of the direct beam (no sample) is shown with the evacuated flight tube (dashed red line) and the flight tube filled with nitrogen at ambient pressure (dashed black line). The blue vertical line delineates the maximal $q$ range in the ptychographic reconstruction.

is fulfilled, where $\langle\bullet\rangle$ denotes the average over the Shannon pixel with solid angle $\Delta \Omega_{d}$ of the feature around the reciprocal space point $\vec{q}$ (cf. Ref. 4). If the background is negligible compared to the scattering from the sample, equation (2) converges to the detection criterion in Ref. 4. A large background, however, requires better statistics (growing linearly with the background).

If the feature is not embedded in a more strongly scattering sample, the detector signal is given by

$$
P(\vec{q})=I_{c} \int_{\Delta \Omega}\left(\left|\psi_{d}(\vec{q})\right|^{2}+b(\vec{q})\right) d \Omega \cdot \Delta t
$$

and the signal-to-noise analysis yields the necessary condition for detecting the feature

$$
I_{c} \Delta t \cdot\left|\psi_{d}\right|^{2} \Delta \Omega_{d} \geq \alpha^{2} \frac{\left|\psi_{d}\right|^{2}+\langle b(\vec{q})\rangle}{\left|\psi_{d}\right|^{2}} .
$$

in analogy to the respective derivation in Ref. 4.

We analyse the signal-to-background for the ptychogram described in section 3 (Fig. 3) and a similar one recorded under the same conditions except for the flight tube being filled with nitrogen at ambient pressure. Fig. 4 shows the azimuthal average over all diffraction patterns of the ptychograms (gray and red solid line, respectively). For the ptychogram that was recorded with the evacuated flight tube, the average diffraction intensity lies well above the background for that case [cf. Fig. 4 (dashed red line)]. As a result, a faithful reconstruction of the ptychogram is possible without taking an incoherent background into account (cf. Fig. 3). ${ }^{\dagger}$ The visibility of features is not significantly affected by the background according to eq. (2).

In the ptychogram recorded with the nitrogen-filled flight tube, the average signal barely emerges from the background in the $q$ range of the ptychogram (left side of the vertical blue line in Fig. 4) and is strongly dominated

\footnotetext{
${ }^{\dagger}$ The background was not included in the ptychographic model for the reconstructions in this article.
} 
a) phase

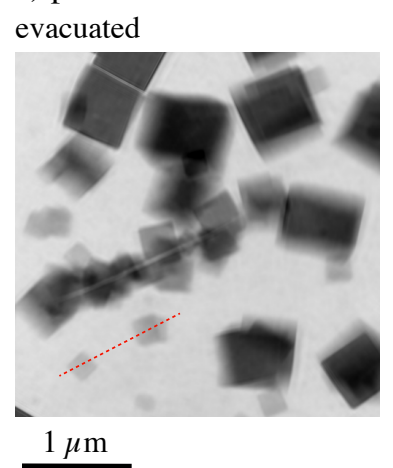

ambient pressure

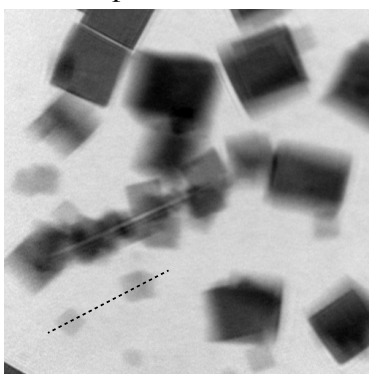

c) line plot of phase

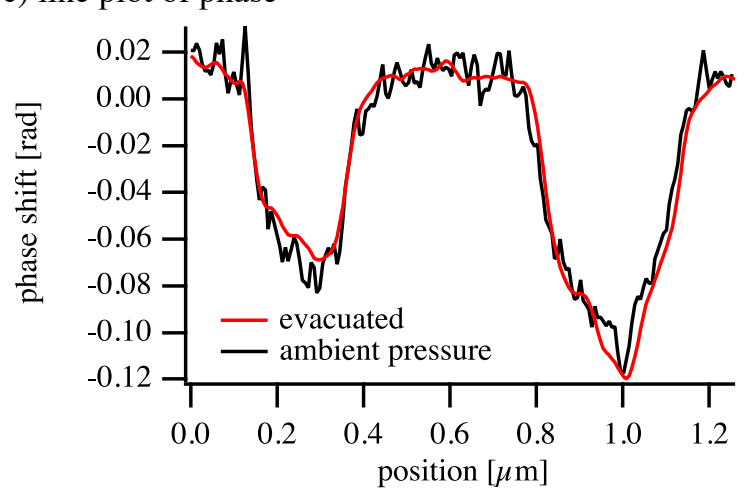

b) amplitude

evacuated

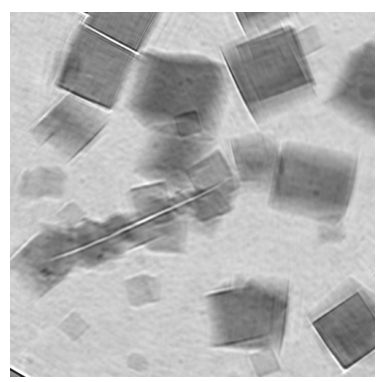

ambient pressure

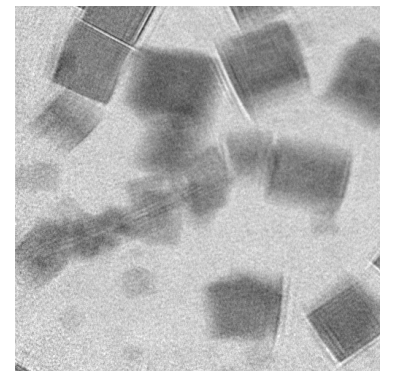

d) ambient pressure diffraction pattern

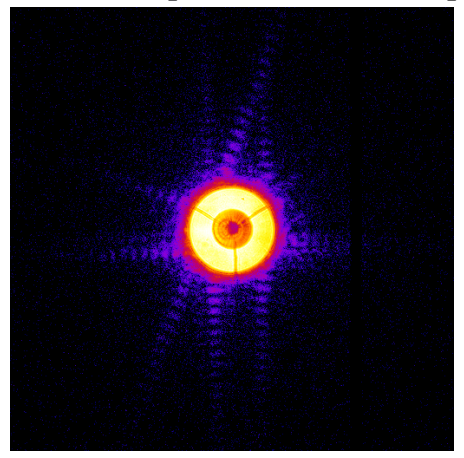

Figure 5. Comparison of the ptychographic data sets with evacuated or ambient pressure flight path, a) phase and b) amplitude. The ptychogram with evacuated flight path is that described in section 3. The other ptychogram was recorded under similar conditions except for the flight tube being filled with nitrogen at ambient pressure. The ptychograms are similar, the ptychogram under ambient conditions being slightly more noisy as illustrated in c), where a section through two particles [dashed lines in a)] is depicted. d) Diffraction pattern from the ambient pressure ptychogram illustrating the strong scattering at the sharp edges of the particles in the sample.

by background for higher $q$. Nevertheless, for the given sample, the reconstruction works very well [Fig. 5a) and b)], since the sharp edges scatter very strongly into a narrow range of reciprocal space, being locally well above the detection limit [cf. Fig. 5d)]. Overall, the reconstruction is noisier with a stronger variation of the background. In addition, edges of more weakly scattering particles appear blurred compared to the ptychogram recorded with evacuated flight path.

For more weakly scattering objects, such as the cluster of nanoparticles shown in Ref. 3, small features can only be resolved with an evacuated flight path. So far, the smallest features that could be detected in the hard X-ray range have a size slightly above $(10 \mathrm{~nm})^{3}$ (cf. Ref. 3). For objects in the single-digit nanometer range, the scattering signal is about three orders of magnitude weaker. To image these small features, the current configuration may not be sufficient and a further reduction of the background may be needed.

\section{CONCLUSION \& OUTLOOK}

In the hard X-ray range, the scattering signal from small objects is very weak, often many orders of magnitude weaker than the direct beam in the forward direction. For ptychography, this implies that in order to be able to resolve small and weakly scattering features, the instrumental background has to be as low as possible. The background can arise from the scattering of optical components along the beam path as well as from residual gas along the optical axis. The strongest contribution comes from scatterers close to the detector, as the solid angle of a detector pixel for these scatterers is relatively large. 
In PtyNAMi, scattering components along the optical axis close to the detector sensor have been minimized. The last optical component is the entrance window (Kapton or diamond) at $>3000 \mathrm{~mm}$ from the sensor. All other optical components are further upstream. The residual gas in the flight tube has a pressure below $1 \times 10^{-3} \mathrm{mbar}$. Upstream of the sample, a pinhole shields the scattering radiation from all upstream components. The pinhole is chosen large enough in order not to cut into the nanofocused X-ray beam. With these measures, a background level is achieved that is about 8 orders of magnitude below the direct beam intensity. While this is sufficient to image objects on the scale of several $10 \mathrm{~nm}$ with high sensitivity, it will not suffice to image single-digit nanometer objects in the future.

Further experimental analysis is needed to determine the origin of the current background level to make the necessary improvements in view of future ptychographic scanning microscopes at fourth generation synchrotron radiation sources. ${ }^{5,42,43}$

\section{ACKNOWLEDGMENTS}

We acknowledge DESY (Hamburg, Germany), a member of the Helmholtz Association HGF, for the provision of experimental facilities. Parts of this research were carried out at beamline P06 of PETRA III.

\section{REFERENCES}

[1] Thibault, P., Dierolf, M., Menzel, A., Bunk, O., David, C., and Pfeiffer, F., "High-resolution scanning x-ray diffraction microscopy," Science 321(5887), 379-382 (2008).

[2] Schropp, A., Hoppe, R., Patommel, J., Samberg, D., Seiboth, F., Stephan, S., Wellenreuther, G., Falkenberg, G., and Schroer, C. G., "Hard x-ray scanning microscopy with coherent radiation: Beyond the resolution of conventional x-ray microscopes," Appl. Phys. Lett. 100, 253112 (2012).

[3] Reinhardt, J., Hoppe, R., Hofmann, G., Damsgaard, C. D., Patommel, J., Baumbach, C., Baier, S., Rochet, A., Grunwaldt, J.-D., Falkenberg, G., and Schroer, C. G., "Beamstop-based low-background ptychography to image weakly scattering objects," Ultramicroscopy 173, 52-57 (2017).

[4] Schropp, A. and Schroer, C. G., "Dose requirements for resolving a given feature in an object by coherent x-ray diffraction imaging," New J. Phys. 12, 035016 (2010).

[5] Schroer, C. G., Agapov, I., Brefeld, W., Brinkmann, R., Chae, Y.-C., Chao, H.-C., Eriksson, M., Keil, J., Gavaldà, X. N., Röhlsberger, R., Seeck, O. H., Sprung, M., Tischer, M., Wanzenberg, R., and Weckert, E., "PETRA IV: The ultra-low emittance source project at DESY," J. Synchrotron Rad. 25(5), 1277-1290 (2018).

[6] Schroer, C. G., Seyrich, M., Kahnt, M., Botta, S., Döhrmann, R., Falkenberg, G., Garrevoet, J., Lyubomirskiy, M., Scholz, M., Schropp, A., and Wittwer, F., "PtyNAMi: Ptychographic nano-analytical microscope at PETRA III: interferometrically tracking positions for 3D x-ray scanning microscopy using a ball-lens retroreflector," Proc. SPIE 10389, 103890E (2017).

[7] Schroer, C. G., Boye, P., Feldkamp, J. M., Patommel, J., Samberg, D., Schropp, A., Schwab, A., Stephan, S., Falkenberg, G., Wellenreuther, G., and Reimers, N., "Hard X-ray nanoprobe at beamline P06 at PETRA III," Nucl. Instrum. Meth. A 616(2-3), 93-97 (2010).

[8] Schroer, C. G., Hönig, S., Goldschmidt, A., Hoppe, R., Patommel, J., Samberg, D., Schropp, A., Seiboth, F., Stephan, S., Schöder, S., Burghammer, M., Denecke, M., Wellenreuther, G., and Falkenberg, G., "Hard x-ray nano-beam characterization by ptychographic imaging," Proc. SPIE 8141, 814103 (2011).

[9] Schroer, C. G., Baumbach, C., Döhrmann, R., Klare, S., Hoppe, R., Kahnt, M., Patommel, J., Reinhardt, J., Ritter, S., Samberg, D., Scholz, M., Schropp, A., Seiboth, F., Seyrich, M., Wittwer, F., and Falkenberg, G., "Hard x-ray nanoprobe of beamline P06 at PETRA III," AIP Conference Proceedings 1741, 030007 (2016).

[10] Reinhardt, J., Schropp, A., Lyubomirskiy, M., Seyrich, M., Brückner, D., Keller, T. F., Vonk, V., Volko, S., Stierle, A., Navickas, E., Fleig, J., and Schroer, C. G., "Hard x-ray resonant ptychography for chemical imaging at the sensitivity limit.," Microscopy and Microanalysis 24, 28-29 (2018).

[11] Schroer, C. G., Kuhlmann, M., Hunger, U. T., Günzler, T. F., Kurapova, O., Feste, S., Frehse, F., Lengeler, B., Drakopoulos, M., Somogyi, A., Simionovici, A. S., Snigirev, A., Snigireva, I., Schug, C., and Schröder, W. H., "Nanofocusing parabolic refractive x-ray lenses," Appl. Phys. Lett. 82(9), 1485-1487 (2003). 
[12] Schroer, C. G., Kurapova, O., Patommel, J., Boye, P., Feldkamp, J., Lengeler, B., Burghammer, M., Riekel, C., Vincze, L., van der Hart, A., and Küchler, M., "Hard x-ray nanoprobe based on refractive x-ray lenses," Appl. Phys. Lett. 87(12), 124103 (2005).

[13] Schroer, C. G., Brack, F.-E., Brendler, R., Hönig, S., Hoppe, R., Patommel, J., Ritter, S., Scholz, M., Schropp, A., Seiboth, F., Nilsson, D., Rahomäki, J., Uhlén, F., Vogt, U., Reinhardt, J., and Falkenberg, G., "Hard x-ray nanofocusing with refractive x-ray optics: full beam characterization by ptychographic imaging," Proc. SPIE 8848, 884807 (2013).

[14] Patommel, J., Klare, S., Hoppe, R., Ritter, S., Samberg, D., Wittwer, F., Jahn, A., Richter, K., Wenzel, C., Bartha, J. W., Scholz, M., Seiboth, F., Boesenberg, U., Falkenberg, G., and Schroer, C. G., "Focusing hard x rays beyond the critical angle of total reflection by adiabatically focusing lenses," Appl. Phys. Lett. 110(10), 101103 (2017).

[15] Kubec, A., Braun, S., Niese, S., Krüger, P., Patommel, J., Hecker, M., Leson, A., and Schroer, C. G., "Ptychography with mulitlayer Laue lenses," J. Synchrotron Rad. 21, 1122-1127 (2014).

[16] Krause, T., Hanke, M., Nicolai, L., Cheng, Z., Niehle, M., Trampert, A., Kahnt, M., Falkenberg, G., Schroer, C. G., Hartmann, J., Zhou, H., Wehmann, H.-H., and Waag, A., "Structure and composition of isolated core-shell (In,Ga)N/GaN rods based on nanofocus x-ray diffraction and scanning transmission electron microscopy," Phys. Rev. Applied 7(2), 024033 (2017).

[17] Dzhigaev, D., Shabalin, A., Stankevic, T., Lorenz, U., Kurta, R. P., Seiboth, F., Wallentin, J., Singer, A., Lazarev, S., Yefanov, O. M., Borgström, M., Strikhanov, M. N., Samuelson, L., Falkenberg, G., Schroer, C. G., Mikkelsen, A., Feidenhans'l, R., and Vartanyants, I. A., "Bragg coherent x-ray diffractive imaging of a single indium phosphide nanowire," J. Opt. 18(6), 064007 (2016).

[18] Schroer, C. G. and Falkenberg, G., "Hard X-ray nanofocusing at low-emittance synchrotron radiation sources," J. Synchrotron Rad. 21(5), 996-1005 (2014).

[19] Rodenburg, J. M. and Faulkner, H. M. L., "A phase retrieval algorithm for shifting illumination," Appl. Phys. Lett. 85(20), 4795-4797 (2004).

[20] Dierolf, M., Menzel, A., Thibault, P., Schneider, P., Kewish, C. M., Wepf, R., Bunk, O., and Pfeiffer, F., "Ptychographic x-ray computed tomography at the nanoscale," Nature 467(7314), 436-440 (2010).

[21] Holler, M., Diaz, A., Guizar-Sicairos, M., Karvinen, P., Färm, E., Härkönen, E., Ritala, M., Menzel, A., Raabe, J., and Bunk, O., "X-ray ptychographic computed tomography at $16 \mathrm{~nm}$ isotropic 3d resolution," Scientific Reports 4, 3857 (2014).

[22] Holler, M., Guizar-Sicairos, M., Tsai, E. H. R., Dinapoli, R., Müller, E., Bunk, O., Raabe, J., and Aeppli, G., "High-resolution non-destructive three-dimensional imaging of integrated circuits," Nature 543, 402-406 (2017).

[23] Beckers, M., Senkbeil, T., Gorniak, T., Reese, M., Giewekemeyer, K., Gleber, S.-C., Salditt, T., and Rosenhahn, A., "Chemical contrast in soft x-ray ptychography," Phys. Rev. Lett. 107(20), 208101 (2011).

[24] Hoppe, R., Reinhardt, J., Hofmann, G., Patommel, J., Grunwaldt, J.-D., Damsgaard, C. D., Wellenreuther, G., Falkenberg, G., and Schroer, C. G., "High-resolution chemical imaging of gold nanoparticles using hard x-ray ptychography," Appl. Phys. Lett. 102(20), 203104 (2013).

[25] Donnelly, C., Guizar-Sicairos, M., Scagnoli, V., Holler, M., Huthwelker, T., Menzel, A., Vartiainen, I., Müller, E., Kirk, E., Gliga, S., Raabe, J., and Heyderman, L. J., "Element-spectific x-ray phase tomography of 3D structures at the nanoscale," Phys. Rev. Lett. 114(11), 115501 (2015).

[26] Donnelly, C., Guizar-Sicairos, M., Scagnoli, V., Gliga, S., Holler, M., Raabe, J., and Heyderman, L. J., "Three-dimensional magnetization structures revealed with X-ray vector nanotomography," Nature 547, 328-331 (2017).

[27] Schropp, A., Boye, P., Feldkamp, J. M., Hoppe, R., Patommel, J., Samberg, D., Stephan, S., Giewekemeyer, K., Wilke, R. N., Salditt, T., Gulden, J., Mancuso, A. P., Vartanyants, I. A., Weckert, E., Schöder, S., Burghammer, M., and Schroer, C. G., "Hard x-ray nanobeam characterization by coherent diffraction microscopy," Appl. Phys. Lett. 96(9), 091102 (2010).

[28] Kewish, C. M., Thibault, P., Dierolf, M., Bunk, O., Menzel, A., Vila-Comamala, J., Jefimovs, K., and Pfeiffer, F., "Ptychographic characterization of the wavefield in the focus of reflective hard X-ray optics," Ultramicroscopy 110(4), 325-329 (2010). 
[29] Kewish, C. M., Guizar-Sicairos, M., Liu, C., Qian, J., Shi, B., Benson, C., Khounsary, A. M., VilaComamala, J., Bunk, O., Fienup, J. R., Macrander, A. T., and Assoufid, L., "Reconstruction of an astigmatic hard x-ray beam alignment of K-B mirrors from ptychographic coherent diffraction data," Opt. Express 18(22), 23420-23427 (2010).

[30] Hönig, S., Hoppe, R., Patommel, J., Schropp, A., Stephan, S., Schöder, S., Burghammer, M., and Schroer, C. G., "Full optical characterization of coherent x-ray nanobeams by ptychographic imaging," Opt. Express 19(17), 16325-16329 (2011).

[31] Vila-Comamala, J., Diaz, A., Guizar-Sicairos, M., Mantion, A., Kewish, C. M., Menzel, A., Bunk, O., and David, C., "Characterization of high-resolution diffractive x-ray optics by ptychographic coherent diffractive imaging," Opt. Express 19(22), 21333-21344 (2011).

[32] Guizar-Sicairos, M., Narayanan, S., Stein, A., Metzler, M., Sandy, A. R., Fienup, J. R., and Evans-Lutterodt, K., "Measurment of hard x-ray lens wavefront aberrations using phase retrieval," Appl. Phys. Lett. 98(11), 111108 (2011).

[33] Schropp, A., Hoppe, R., Meier, V., Patommel, J., Seiboth, F., Lee, H. J., Nagler, B., Galtier, E. C., Arnold, B., Zastrau, U., Hastings, J. B., Nilsson, D., Uhlen, F., Vogt, U., Hertz, H. M., and Schroer, C. G., "Full spatial characterization of a nanofocused x-ray free-electron laser beam by ptychographic imaging," Scientific Reports 3, 1633 (2013).

[34] Seiboth, F., Scholz, M., Patommel, J., Hoppe, R., Wittwer, F., Reinhardt, J., Seidel, J., Knaut, M., Jahn, A., Richter, K., Bartha, J. W., Falkenberg, G., and Schroer, C. G., "Hard x-ray nanofocusing by refractive lenses of constant thickness," Appl. Phys. Lett. 105(13), 131110 (2014).

[35] Morgan, A. J., Prasciolu, M., Andrejczuk, A., Krzywinski, J., Meents, A., Pennicard, D., Graafsma, H., Barty, A., Bean, R. J., Barthelmess, M., Oberthuer, D., Yefanov, O., Aquila, A., Chapman, H. N., and Bajt, S., "High numerical aperture multilayer Laue lenses," Scientific Reports 5, 09892 (2015).

[36] Seiboth, F., Schropp, A., Scholz, M., Wittwer, F., Rödel, C., Wünsche, M., Ullsperger, T., Nolte, S., Rahomäki, J., Parfeniukas, K., Giakoumidis, S., Vogt, U., Wagner, U., Rau, C., Boesenberg, U., Garrevoet, J., Falkenberg, G., Galtier, E. C., Lee, H. J., Nagler, B., and Schroer, C. G., "Perfect X-ray focusing via fitting corrective glasses to aberrated optics," Nature Communications 8, 14623 (2017). doi: 10.1038/ncomms14623.

[37] Howells, M. R., Beetz, T., Chapman, H. N., Cui, C., Holton, J. M., Jacobsen, C. J., Kirz, J., Lima, E., Marchesini, S., Miao, H., Sayre, D., Shapiro, D. A., Spence, J. C. H., and Starodub, D., "An assessment of the resolution limitation due to radiation-damage in x-ray diffraction microscopy," J. Electron Spect. 170(13), 4-12 (2009).

[38] Bernert, C., Hoppe, R., Wittwer, F., Woike, T., and Schroer, C. G., "Phychographic analysis of the photorefractive effect in $\mathrm{LiNbO}_{3}$ :Fe," Opt. Express 25(25), 31640 (2017).

[39] Chang, H., Enfedaque, P., Zhang, J., Reinhardt, J., Enders, B., Yu, Y.-S., Shapiro, D., Schroer, C. G., Zeng, T., and Marchesini, S., "Advanced denoising of x-ray ptychography," Optics Express 27(8), 10395-10418 (2019).

[40] Kränzlin, N., Ellenbroek, S., Durán-Martín, D., and Niederberger, M., "Liquid-phase deposition of freestanding copper foils and supported copper thin films and their structuring into conducting line patterns," Angew. Chem. Int. Ed. 51, 4743-4746 (2012).

[41] Kränzlin, N., van Beek, W., Niederberger, M., and Koziej, D., "Mechanistic studies as a tool for the design of copper-based heterostructures," Adv. Mater. Interfaces 2, 1500094 (2015).

[42] Tavares, P. F., Al-Dmour, E., Andersson, Å., Cullinan, F., Jensen, B. N., Olsson, D., Olsson, D. K., Sjöström, M., Tarawneh, H., Thorin, S., and Vorozhtsov, A., "Commissioning and first-year operational results of the MAX IV 3 GeV ring," J. Synchrotron Rad. 25, 1291-1316 (2018).

[43] Raimondi, P., "ESRF-EBS: The extremely brilliant source project," Synchr. Rad. News 29(6), 8-15 (2016). 